\title{
Superior Vena Cava Syndrome
}

National Cancer Institute

\section{Source}

National Cancer Institute. Superior Vena Cava Syndrome. NCI Thesaurus. Code C3396.

Obstruction of the blood flow in the superior vena cava caused by a malignant neoplasm, thrombosis, or aneurysm. It is a medical emergency requiring immediate treatment. Signs and symptoms include swelling and cyanosis of the face, neck, and upper arms, cough, orthopnea, and headache. 